\title{
Kampo medicines improved blood test and QOL in two vasculitis cases of Churg-Strauss syndrome and Henoch-Shönlein purpura after inadequate treatment with conventional therapies
}

This article was published in the following Dove Press journal:

International Journal of General Medicine

20 February 2010

Number of times this article has been viewed

\author{
Yasuyo Hijikata' \\ Yumiko Ikemoto ${ }^{2}$ \\ Urara Kohdera ${ }^{3}$ \\ 'Toyodo Hijikata Clinic, Osaka, \\ Japan; ${ }^{2}$ St Mary's Hospital, Himeji, \\ Japan; ${ }^{3}$ Nakano Children's Hospital, \\ Osaka, Japan
}

Correspondence: Yasuyo Hijikata Toyodo Hijikata Clinic, 3-I I-29, Kasuga, Ibaraki, Osaka, Japan

Tel +8I 726273756

Fax +8I 726273991

Email hijikata@hcn.zaq.ne.jp
Objective: Based on the tenets of traditional Chinese medicine (TCM) theory, Kampo medicines were selected and applied to two cases of Churg-Strauss syndrome and Henoch-Shönlein purpura. Two vasculitis syndrome patients exhibited persistent symptoms and abnormal blood tests after treatment with conventional therapies.

Methods: As the two cases had "blood stagnation" and "damps and heat" and one had a "yang deficiency" in terms of TCM theory, we applied certain selected Kampo medicines.

Results: In case 1, the patient presented with hypereosinophilia, venous thrombosis, pulmonary infarction, decreased platelet count, ulner nerve palsy and Raynaud's phenomena, which led to a diminished quality of life. After starting the Kampo medicines, the patient improved quickly and recovered within 11 months. In case 2, persistent purpura, abdominal pain, and bloody feces quickly improved and disappeared after Kampo treatment. After starting the Kampo medicines, prednisolone was stopped at 21 days without any sign of relapse to date.

Conclusion: Kampo medicines helped clear the persistent abnormal symptoms and laboratory findings of vasculitis syndromes, Churg-Strauss syndrome and Henoch-Shönlein purpura, which had responded inadequately to the conventional therapies administered.

Keywords: Kampo medicine, vasculitis, Churg-Strauss syndrome, Henoch-Shönlein purpura

\section{Introduction}

Churg-Strauss Syndrome (CSS) and Henoch-Shönlein purpura (HSP) belong to the vasculitis syndromes, in which inflammation and blood vessel damage are common. Since the blood vessels are normally responsible for sending adequate nourishment to the bodily tissues and the interior of these vessels is easily damaged, various pathologies result from such vessel damage. ${ }^{1}$

CSS is uncommon, and necrotizing vasculitis involves small and medium-sized arteries, capillaries, veins, and venules. The histopathologic feature of CSS is granulomatous reactions that may be present in the tissues or even within the walls of the vessels themselves. The process can occur in any organ in the body, but lung involvement is predominant, with manifestations in the skin, cardiovascular system, kidney and peripheral nervous system, and occasionally the gastrointestinal tract. Although the pathogenesis is uncertain, it has a strong association with asthma and its clinicopathologic manifestations, including eosinophilia, granuloma, and vasculitis. $^{2}$ 
HSP is usually seen in children, and is characterized by palpable purpura, arthralgias, gastrointestinal signs and symptoms, and glomerulonephritis. It is a small-vessel vasculitis. The presumptive pathogenic mechanism is immune-complex deposition. Diagnosis of HSP is based on clinical signs and symptoms. A skin biopsy specimen is useful to confirm leukocytoclastic vasculitis with IgA and C3 deposition by immunofluorescence. ${ }^{3}$

These diseases are reported to exhibit a good response to corticosteroid hormones. However, there are patients with CSS or HSP who do not completely recover and suffer from an incomplete remission state which degrades their quality of life (QOL).

The patient with CSS improved to some degree, with the application of conventional therapies in a university hospital, at the acute stage. However at the chronic stage symptoms included hypereosinophilia, venous thrombosis, pulmonary infarction, decreased platelet count, and Raynaud's phenomena which did not improve and the patient had persistent coldness of the limbs and an unpleasant facial flush in a warm environment, which lowered QOL drastically.

The patient with HSP showed improvement with conventional therapies, mainly steroidal hormones. However, his condition worsened soon after a tapering down of the prednisolone dose which had been repeated.

From the perspective of traditional Chinese medicine (TCM) theory, we diagnosed the pathogenesis of these diseases as related to "static blood"4 and in the case of the inflammation of the vasculitis, as related to "dampnessheat", 5,6 mainly in the morbid vessels, and also, in case, 1 , yang deficiency (coldness of finger and limbs and/or being sensitive to coldness). ${ }^{7}$ We treated the patients with Kampo medicines which are traditionally used to "activate blood and resolve stasis" and "clear heat and drain dampness" and also, only in case 1, added a Kampo medicine which warms the channels or meridians, ${ }^{9}$ dissipates cold, ${ }^{10}$ and unblocks the blood vessels. These treatments helped bring about prompt recovery and a return to a normal QOL.

\section{Case reports}

\section{Case I:A 13-year-old boy with CSS}

The patient's complaints were persistent hypereosinophilia, left femoral deep venous thrombosis, pulmonary infarction, decreased platelet count, Raynaud's phenomena since the start of disease, facial hot flushes in warm environments, ulnar nerve palsy, and pain with edema of the legs after extended walking, despite the conventional therapies administered.
For three years prior to admission to the university hospital with malaise, petechiae, and purpura, the patient had been suffering from allergic rhinitis. He was diagnosed as having CSS with hypereosinophilia, vasculitis with extravascular eosinophils accumulation, diffuse pulmonary infiltrate, and ulnar nerve palsy. Furthermore he had left femoral deep venous thrombosis and pulmonary infarction. On the 5th day after hospitalization for suspected pneumonia with septic shock, he received pulse doses of methylprednisolone and gabexate mesilate. Following this treatment the fever, chest pain and the diffuse pulmonary infiltrate disappeared along with normalization of eosinophil count (Figure 1). For the persistent left femoral deep venous thrombosis and pulmonary infarction, he was given heparin and warfarin with prednisolone. which continued to be administered even after discharge from the hospital (Figures 2 and 3). ${ }^{11}$

The pulmonary segmental shadow caused by pulmonary infarction disappeared after six months. The left femoral deep venous thrombosis and ulnar nerve palsy disappeared after nine months (Figures 1-3).

At 81 days after discharge from the hospital, the Kampo medicine "Saireito" (Chi ling tang: in Chinese) (TJ 114) was started (two-packs/day: a normal dose for a child) to prevent the side effects of prednisolone. ${ }^{12} \mathrm{TJ}$ indicates that this is a product of Tsumura Co (Akasaka, Minatoku, Tokyo, Japan).

The medical practitioner decided to apply Kampo medicine because there were signs of hypereosinophilia remaining seven months after discharge from the hospital, with an unstable platelet count, femoral deep vein thrombosis, ulnar nerve palsy, Raynaud's phenomena, facial hot flushes in a warm environment, leg pain with edema after extended walking, and coldness of the limbs accompanied by reduced QOL. The patient visited the Toyodo Hijikata Clinic on the 224th day after discharge from the hospital.

From the standpoint of TCM theory, he was diagnosed as having "static blood" based on thrombosis and infarction of the blood vessels and dark vena sublingualis, as well as "dampness-heat" $" 5,6$ in the affected vessels, based on vasculitis, and as having a "Yang deficiency" based on cold limbs, sensitivity to cold, and Raynaud's phenomenon. Accordingly, we prescribed the decoction of Tokishigyakuka-gosyuyusyokyoto for the Yang deficiency to warm the channels, disperse cold, nourish the blood and unblock the blood vessels (Table 1) ${ }^{13}$ For "blood stasis", Botanpi (mutanpi), ${ }^{14}$ Tonin (tauren), ${ }^{15}$ Syachu (zhe chong), ${ }^{16}$ and Suitetsu (shui zhi) ${ }^{17}$ were added. For "dampness-heat", Orengedokuto (TJ-15) "18-20 $^{17}$ (Table 2) was prescribed. 

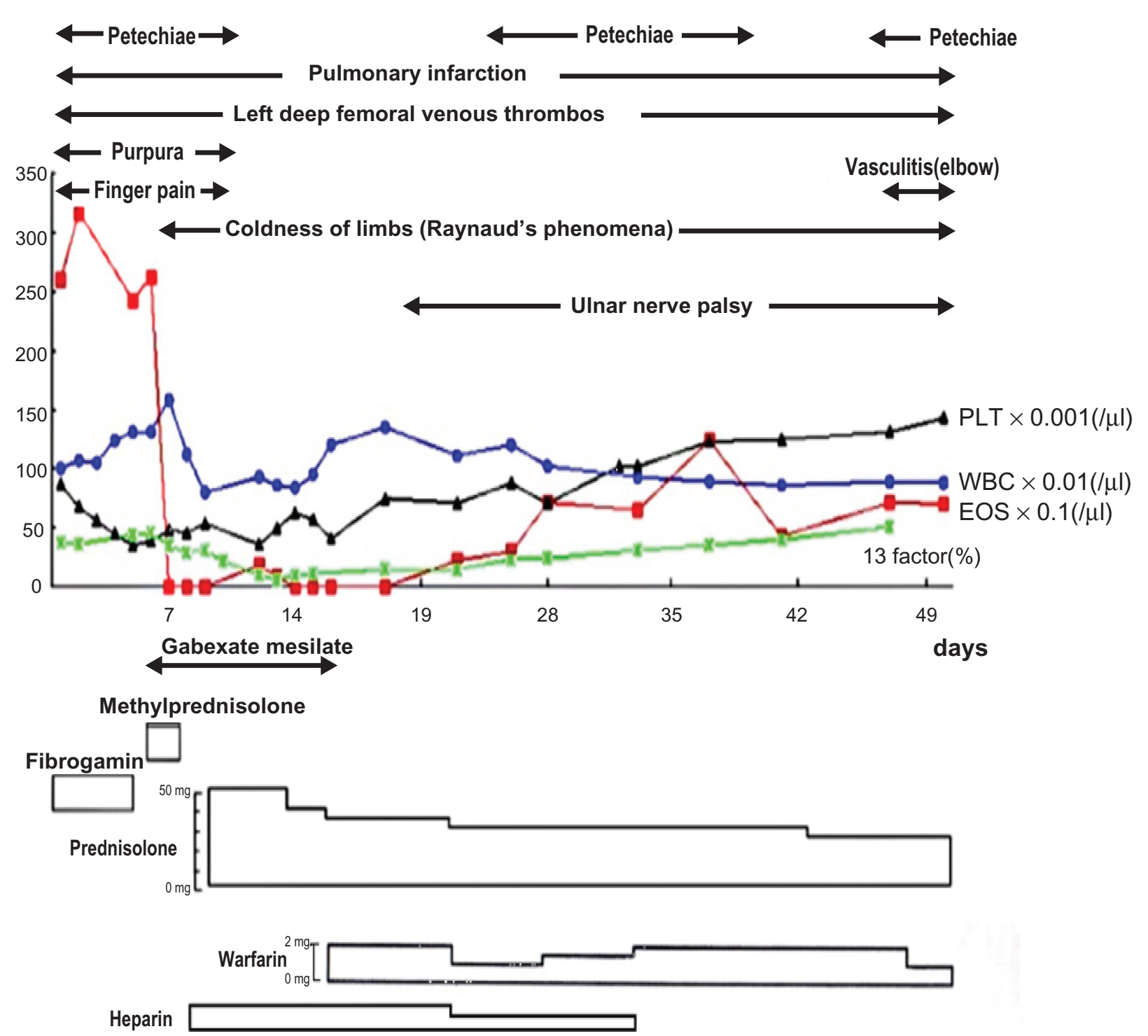

Figure I Clinical course during hospitalization of Case I.

After prescribing Kampo medicines, the Raynaud's phenomena, cold and painful limbs, and abnormal platelet count quickly normalized, and hypereosinophilia gradually improved to a normal level, with transient deterioration, over a period of 10 months. The left femoral venous thrombosis and ulnar nerve palsy disappeared after nine months (Figure 3).

The rationale for why these Kampo medicines were applied is related to the activities of these respective herbs composing Kampo medicines as seen in Tables 1-4.

\section{Case 2:A 2-year-old boy}

Since the middle of April 2000, the patient had been suffering from a common cold. On the 25th of April, petechiae appeared on the right leg and spread to the left leg and both forearms.
Abdominal pain with watery diarrhea, bloody stools, and knee joint pain were noted, with the latter appearing and recovering spontaneously. As the purpura became aggravated, he was hospitalized and diagnosed with HSP.

He was treated with prednisolone successfully for the abdominal pain and bloody stools, but the symptoms reappeared with the tapering of the drug (Figure 4). The family of the patient requested treatment with Kampo medicine.

Based on TCM theory he was diagnosed as having "blood stagnation" with purpura, ${ }^{4}$ as having Yin deficiency with Yang hyperactivity ${ }^{21}$ based on a rapid pulse (90/min) and excitability, along with "dampness-heat" based on the existence of vasculitis and preference for cold rather than hot food. We prescribed Keishi-bukuryogan (Guizhifulinwan) 


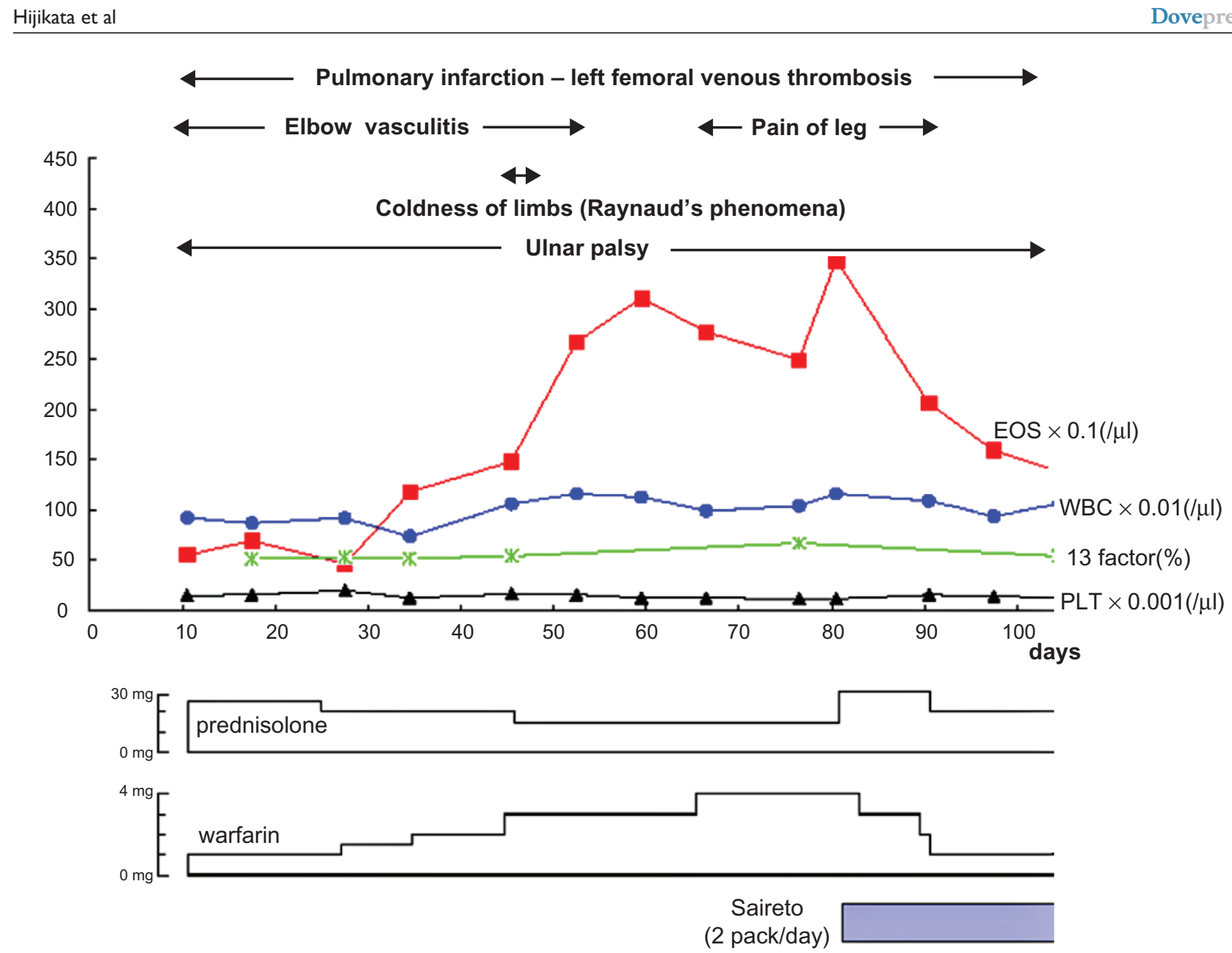

Figure 2 Clinical course after discharge from the hospital of Case I.

(TJ 25) for "blood stagnation"22 (Table 3). Sanmotsuogonto (Sanwuhuangqintang) (TJ 121) (Table 4) ${ }^{19,23-25}$ for Yin deficiency with Yang hyperactivity ${ }^{21}$ and Orengedokuto (Huanlienjiedutang) (TJ 15) for "clearing dampness-heat" (Table 2). ${ }^{18}$

As shown in Figure 4, in spite of the tapering down of the prednisolone, soon after the administration of the Kampo medicines, the purpura and abdominal pain drastically decreased, and the bloody stool subsided. Despite the fact that he stopped the prednisolone three weeks after starting the Kampo medicines, his improved condition continued, leading to discharge from the hospital.

\section{Discussion}

In Case 1, the platelet decrease might be the result of a pulmonary infarction. The prednisolone pulse therapy may have aggravated the infarction. It is not clear whether his mother's toxoplasmosis infection during pregnancy influenced his constitution.
In CSS with eosinophilia and systemic vasculitis, "blood stagnation" is present due to the pulmonary infiltrate of eosinophilia caused by vasculitis. As mentioned above, botanpi, tonin, syachu, and suitetsu resolved the blood stagnation and accelerated the blood flow. ${ }^{14-17}$ Tokisigyakukagosyuyusyokyoto (Tangukuei decoction for frigid extremities plus evodia and fresh ginger), ${ }^{13}$ which in TCM warm the channels, disperse cold, and unblock blood vessels, were given.

For inflammation, "dampness-heat" in TCM, Orengedokuto, which traditionally relieves heat and dampness, was applied. These formulas and remedies are considered to work synergistically to prompt recovery.

With Case 2, as shown in Case 1, vasculitis has the characteristic of "heat and dampness", for which Orengedokuto was applied. Deposits of immune complex in the blood vessels or purpura mean blood stagnation in TCM, for which Keishibukuryogan was applied (Table 3). The fact the patient preferred cold drink, and was feverish with 


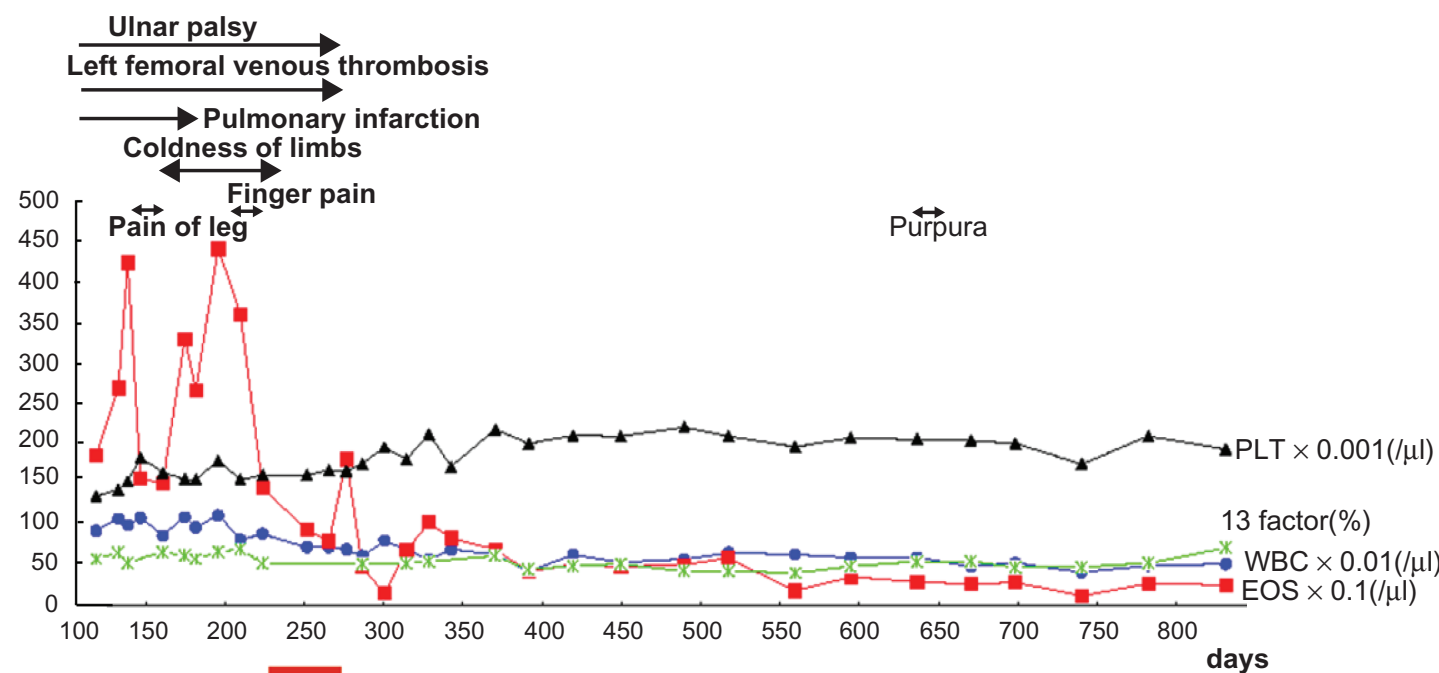

Tokishigyakukagoshuyushokyoto

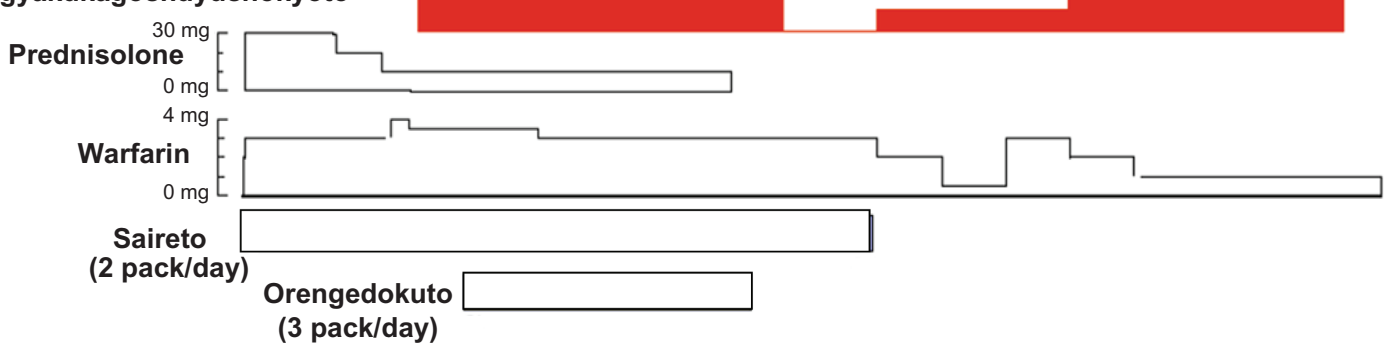

Figure 3 Clinical course after discharge from the hospital (continued from Figure 2) of case I.

Table I Composition of herbs in Tokishigyakukagosyuyusyokyoto (当帰四逆加吳茱䔾生姜湯)' and the main function of respective herbs

\begin{tabular}{ll}
\hline $\begin{array}{l}\text { Name of the herb (Japanese: } \\
\text { Chinese), N (g/day) }\end{array}$ & Main function \\
\hline $\begin{array}{l}\text { Radix Angelicae Sinensis } \\
\text { (Toki: dang gui), 3 }\end{array}$ & Tonifies and invigorates the blood \\
Radix Paeoniae Lactiflorae & Strengthens the tonifying action of \\
(Syakuyaku: bai shao), 3 & Toki \\
Ramulus Cinnamomi Cassiae & Warms the meridian and disperses \\
(Keishi: gui zhi), 3 & cold \\
Herba cum Radice Asari & Disperses both internal and \\
(Saishin: xi xin), 2 & external cold \\
Radix Glycyrrhizae Uralensis & Auguments the Qi and strengthens \\
(Kanzo: gan cao), I & the Spleen \\
Fructus Zizyphi Jujubae & Auguments the Qi and strengthens \\
(Taiso: da cao), 5 & the Spleen \\
Caulis Mutong & Facilitates the flow in the channels \\
(Mokutsu: mu tong), 3 & and vessels \\
Fructus Evodiae Rutaecarpae & Warms the middle, disperses cold, \\
(Gosyuyu: Wu zhu yu), 2 & promotes the movement of Qi \\
Zingiberis Officinalis Recens & Release the exteriors and disperses \\
(dried Syokyo: heng jiang), I & cold \\
\hline
\end{tabular}

Notes: 'Tangkuei decoction for frigid extremities plus evodia and fresh ginger (US name), Dang gui si ni jia wu zhu yu sheng jiang tang (Chinese name [pinyin]). ${ }^{13}$ $\mathrm{N}=$ mean weight of dried herbs ( $\mathrm{g} / \mathrm{day})$. For Case $\mathrm{I}, 2 \mathrm{~g}$ of Botanpi and Tonin and $0.5 \mathrm{~g}$ of Shachu and Suitetsu were added to the above prescriptions to decoct with water. Japanese and Chinese pronunciation are expressed in parenthesis. a rapid pulse rate suggested Yin deficiency with internal heat, ${ }^{26}$ for which Sanmotsuogonto was prescribed (Table 4). ${ }^{23}$ These prescriptions are also supposed to work synergistically so as to affect a quick recovery.

These cases with systemic vasculitis had "blood stagnation" and "dampness-heat" in terms of TCM. Properly selected remedies for "blood stagnation" and "heat and dampness" seemed to help achieve patient improvement.

Table 2 Composition of herbs in Orengedokuto (黄連解毒湯) and the main function of respective herbs

\begin{tabular}{ll}
\hline $\begin{array}{l}\text { Name of the herb } \\
\text { (Japanese: Chinese), N }\end{array}$ & Main function \\
\hline $\begin{array}{l}\text { Rhizoma Coptidis } \\
\text { (Oren: huang lian), } 2\end{array}$ & Clears heat and dries dampness \\
$\begin{array}{l}\text { Radix Scutellariae } \\
\text { (Ogon: huang qin), } 3\end{array}$ & Clears heat and dries dampness \\
$\begin{array}{l}\text { Cortex Phellodendri } \\
\text { (Obaku: huang bai), I.5 }\end{array}$ & Clears heat and dries dampness \\
$\begin{array}{l}\text { Fructus Gardeniae } \\
\text { Jasminoidis (Shishi: zhi zi), } 2\end{array}$ & Clears heat and eliminates irritability \\
\hline
\end{tabular}

Notes: Coptis decoction to relieve toxicity (US name ${ }^{18}$ ), Huang lian jie du tang (Chinese name).

$\mathrm{N}$ (see Table I). 

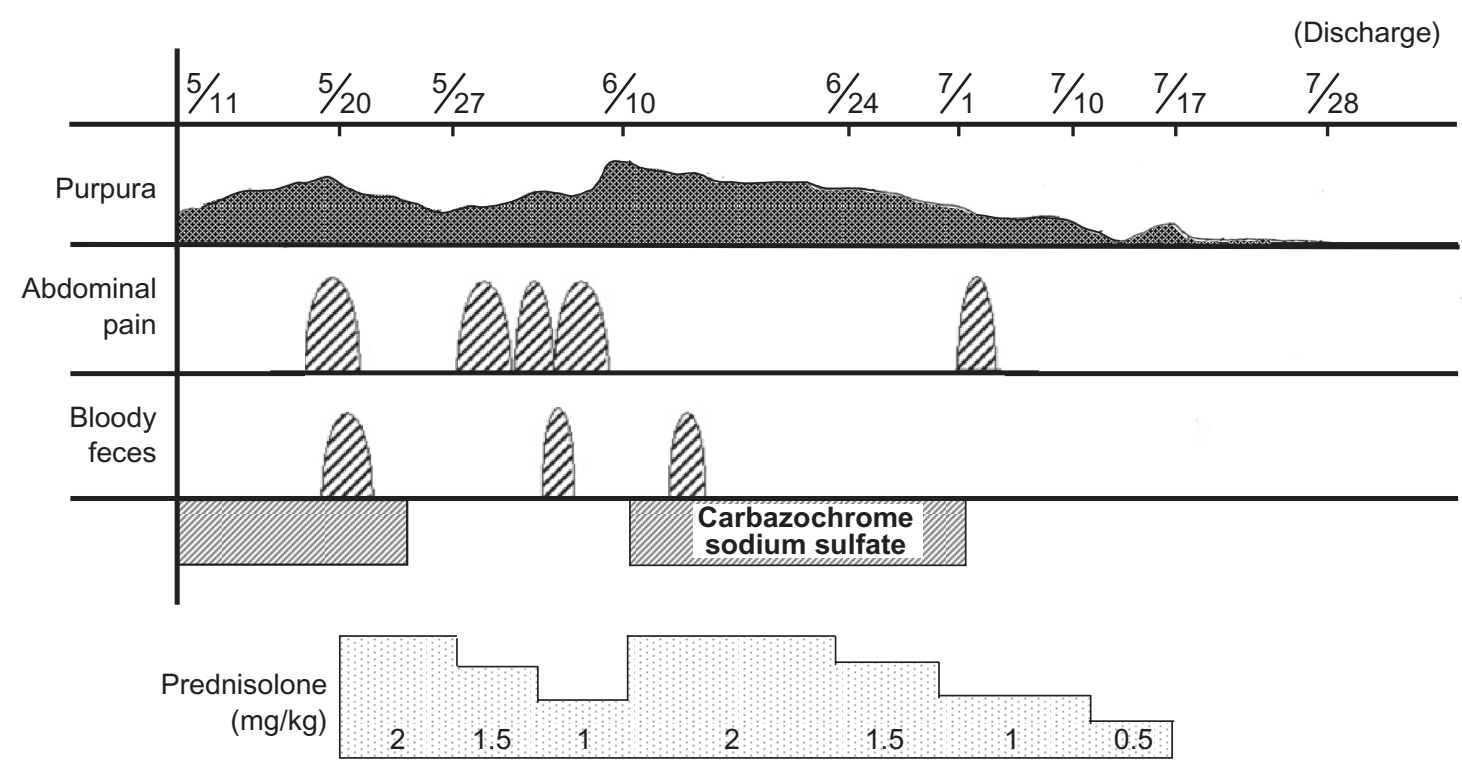

kampo medicine

Orengedokuto, Keishibukuryogan, Sanmotsuogonto

Figure 4 Clinical course of case 2 during hospitalization.

In Japan many doctors treat diseases which are not treatable with conventional therapies with Kampo medicines or TCM. Further investigation is needed to give better insight into which of these medicines should be incorporated into normal practice.

\section{Disclosures}

The authors report no conflicts of interest in this work.

Table 3 Composition of herbs in Keshibukuryogan(桂枝获苓丸)* and the main functions of respective herbs

\begin{tabular}{ll}
\hline $\begin{array}{l}\text { Name of herbs (Japanese: } \\
\text { Chinese), N (g/day) }\end{array}$ & Main function \\
\hline $\begin{array}{l}\text { Ramulus Cinnamomi Cassiae } \\
\text { (Keishi: gui zhi), } 3\end{array}$ & $\begin{array}{l}\text { Unblocks the blood vessels and } \\
\text { promotes blood circulation }\end{array}$ \\
$\begin{array}{l}\text { Sclerotium Poriae Cocos } \\
\text { (Bukuryo: fu ling), } 3\end{array}$ & $\begin{array}{l}\text { Transforms phlegm and promotes } \\
\text { urination, nourishes the Qi and } \\
\text { calms the spirit }\end{array}$ \\
Radix Paeoniae & $\begin{array}{l}\text { Relaxes spasms and alleviates } \\
\text { abdominal pain }\end{array}$ \\
(Syakuyaku: shao yao), 3 & $\begin{array}{l}\text { Cools and invigorates the blood } \\
\text { Cortex Moutan Radicis }\end{array}$ \\
(Botanpi: mu dan pi), 3 & and dispels blood stasis \\
Semen Persicae & Invigorates the blood and dispels \\
(Tonin: tao ren), 3 & blood stasis \\
\hline
\end{tabular}

Notes: "Cinnamon twig and Poria pill (US name), Gui zhi fu ling wan (Chinese name). ${ }^{22}$ $\mathrm{N}$ (see Table I).
Table 4 Composition of herbs in Sanmotsuogonto (三物黄芩湯)* and the main function of respective herbs

\begin{tabular}{|c|c|}
\hline $\begin{array}{l}\text { Name of herbs (Japanese: } \\
\text { Chinese), } N \text { (g/day) }\end{array}$ & Main function in human \\
\hline $\begin{array}{l}\text { Radix Scutellariae } \\
\text { (Ogon: huam qin) }^{19,6}\end{array}$ & Clears heat and dries dampness \\
\hline $\begin{array}{l}\text { Radix Rehmanniae } \\
\text { (Shojio: sheng di huang) }{ }^{24,3}\end{array}$ & $\begin{array}{l}\text { Clears heat and cools blood. Nourishes } \\
\text { the Yin and generates fluids }\end{array}$ \\
\hline 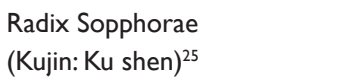 & $\begin{array}{l}\text { Clears heat and dries dampness. Kills } \\
\text { parasites and stops dampness and itching }\end{array}$ \\
\hline
\end{tabular}

Notes: *Three herbs Baikal Skullcap decoction (alternative Japanese name). ${ }^{23}$

\section{References}

1. Kasper DL, Braunwald E, Fauci AS, Hauser SL, Longo DL, Jameson JL. In: Harrison's Principles of Internal Medicine (16th Edition). New York, NY: McGraw-Hill; 2005:2007.

2. Kasper DL, Braunwald E, Fauci AS, Hauser SL, Longo DL, Jameson JL. In: Harrison's Principles of Internal Medicine (16th Edition). New York, NY: McGraw-Hill; 2005:2007-2008.

3. Kasper DL, Braunwald E, Fauci AS, Hauser SL, Longo DL, Jameson JL. In: Harrison's Principles of Internal Medicine (16th Edition). New York, NY: McGraw-Hill; 2005:2007.

4. World Health Organization. TERMINOLOGIES/Cause of Disease (1.6.0): static blood (1.6.84). WHO International Standard Terminologies on Traditional Medicine in the Western Pacific Region. Geneva, Switzerland: World Health Organization; 2007:45.

5. World Health Organization. TERMINOLOGIES/Cause of Disease (1.6.0): dampness (1.6.14). WHO International Standard Terminologies on Traditional Medicine in the Western Pacific Region. Geneva, Switzerland: World Health Organization; 2007:40. 
6. World Health Organization. TERMINOLOGIES/Cause of Disease (1.6.0): heat (1.6.17). WHO International Standard Terminologies on Traditional Medicine in the Western Pacific Region. Geneva, Switzerland: World Health Organization; 2007:40.

7. World Health Organization. TERMINOLOGIES/Mechanism of Disease (1.7.0): yang deficiency with yin exuberance (1.7.31). WHO International Standard Terminologies on Traditional Medicine in the Western Pacific Region. Geneva, Switzerland: World Health Organization; 2007:48.

8. World Health Organization. TERMINOLOGIES/Method of Treatment (4.2.0): clear heat and drain dampress (4.2.47). WHO International Standard Terminologies on Traditional Medicine in the Western Pacific Region. Geneva, Switzerland: World Health Organization; 2007:209.

9. World Health Organization. TERMINOLOGIES/Method of Treatment (4.2.0): warm the meridian (4.2.157). WHO International Standard Terminologies on Traditional Medicine in the Western Pacific Region. Geneva, Switzerland: World Health Organization; 2007:217.

10. World Health Organization. TERMINOLOGIES/Method of Treatment (4.2.0): dissipate cold (4.2.15).WHO International Standard Terminologies on Traditional Medicine in the Western Pacific Region. Geneva, Switzerland: World Health Organization; 2007:206.

11. Ikemoto Y, Kohdera U, Uraoka M, Teraguchi M, Okamura A, Kobayashi Y. Pulmonary infarction and deep venous thrombosis in a 13-year-old boy with Churg-Strauss syndrome. Pediatr Int. 2001;43:441-443.

12. Kimura K, Nanba S, Tojo A, Matsuoka H, Sugimoto T. Effects of saireito on the relapse of steroid-dependent nephrotic syndrome. Am J Chin Med. 1990;18(1-2):45-50.

13. Bensky D, Barolet R. Formulas that warm the channels and disperse cold. In: Scheid V, Bensky D, Ellis A, Barolet R, editors. Chinese Herbal Medicine: Formulas and Strategies. Vista, CA: Eastland Press; 1990:216-217.

14. Herbs that clear heat. In: Bensky D, Clavey S, Stoger E, Gamble A, editors. Chinese Herbal Medicine: Materia Medica. 3rd Edition. Vista, CA: Eastland Press; 2004:126-128.

15. Herbs that regulate the blood. In: Bensky D, Clavey S, Stoger E, Gamble A, editors. Chinese Herbal Medicine: Materia Medica. 3rd Edition. Vista, CA: Eastland Press; 2004:624-627.
16. Herbs that regulate the blood. In: Bensky D, Clavey S, Stoger E, Gamble A, editors. Chinese Herbal Medicine: Materia Medica. 3rd Edition. Vista, CA: Eastland Press; 2004:655-656.

17. Herbs that regulate the blood. In: Bensky D, Clavey S, Stoger E, Gamble A, editors. Chinese Herbal Medicine: Materia Medica. 3rd Edition. Vista, CA Eastland Press; 2004:656-657.

18. Bensky D, Barolet R. Formulas that clear heat and relieve toxicity. In: Scheid V, Bensky D, Ellis A, Barolet R, editors. Chinese Herbal Medicine: Formulas and Strategies. Vista, CA: Eastland Press; 1990:78-79.

19. Herbs that clear heat. In: Bensky D, Clavey S, Stoger E, Gamble A, editors. Chinese Herbal Medicine: Materia Medica. 3rd Edition. Vista CA: Eastland Press; 2004:130-141.

20. Herbs that clear heat. In: Bensky D, Clavey S, Stoger E, Gamble A, editors. Chinese Herbal Medicine: Materia Medica. 3rd Edition. Vista, CA: Eastland Press; 2004:95-98.

21. World Health Organization. TERMINOLOGIES/Mechanism of Disease (1.7.0): yin deficiency with yang hyperactivity (1.7.24). WHO International Standard Terminologies on Traditional Medicine in the Western Pacific Region. Geneva, Switzerland: World Health Organization; 2007:47.

22. Bensky D, Barolet R. Formulas that warm the menses and dispel blood statis. In: Scheid V, Bensky D, Ellis A, Barolet R, editors. Chinese Herbal Medicine: Formulas and Strategies. Vista, CA: Eastland Press; 1990:322-323.

23. Yasui H. Appendix. Composition and indications of 148 prescriptions: Three herbs baikal skullcap decoction. Kampo Acupuncture and Integrative Medicine. 2005; 1(Special Edition): 99.

24. Herbs that clear heat. In: Bensky D, Clavey S, Stoger E, Gamble A, editors. Chinese Herbal Medicine: Materia Medica. 3rd Edition. Vista, CA: Eastland Press; 2004:120-123.

25. Herbs that clear heat. In: Bensky D, Clavey S, Stoger E, Gamble A, editors. Chinese Herbal Medicine: Materia Medica. 3rd Edition. Vista, CA: Eastland Press; 2004:144-146.

26. World Health Organization. TERMINOLOGIES/Mechanism of Disease (1.7.0): yin deficiency with infernal heat (1.7.23). WHO International Standard Terminologies on Traditional Medicine in the Western Pacific Region. Geneva, Switzerland: World Health Organization; 2007:47.
International Journal of General Medicine

\section{Publish your work in this journal}

The International Journal of General Medicine is an international, peer-reviewed open-access journal that focuses on general and internal medicine, pathogenesis, epidemiology, diagnosis, monitoring and treatment protocols. The journal is characterized by the rapid reporting of reviews, original research and clinical studies across all disease areas.

\section{Dovepress}

A key focus is the elucidation of disease processes and management protocols resulting in improved outcomes for the patient.The manuscript management system is completely online and includes a very quick and fair peer-review system. Visit http://www.dovepress.com/ testimonials.php to read real quotes from published authors. 